\title{
THE SECOND DUAL OF THE SPACE OF CONTINUOUS FUNCTIONS, II
}

\author{
BY \\ SAMUEL KAPLAN( ${ }^{(1)}$
}

1. Introduction. In this paper we continue our study [10] of the Banach lattice $C$ of real continuous functions on a compact Hausdorff space, and of its first and second duals, $L$ and $M$. The principal new tool is the extensive theory developed by Nakano [12] of the linear functionals on a vector lattice which are continuous with respect to (order) convergence. Through them a richer duality is obtained and a deeper analysis of the structure of the above spaces. Because of the importance of Nakano's results, not only for the present paper but also for a succeeding one on locally compact spaces, and because the particular results we want are interwoven over more than a hundred pages of Nakano's book with a wealth of other material, we devote $\$ 3$ to a self-contained exposition of these results.

In $\$ 4$ we present the applications of the Nakano theory to our spaces. An immediate one is that the smallest subset of $M$ containing $C$ which is closed under (order) convergence is $M$ itself. This in turn gives us that $C$ is dense in $M$ under $|w|(M, L)$, a result which required an involved proof in our first paper. Other results: the duality of $L$ and $M$ with respect to the above discussed (order) continuity; the reflexivity with respect to this same (order) continuity of every (order) closed sub-vector-lattice of $L$ or $M$; the property that every (order) closed sub-vector-lattice of $L$ or $M$ is closed under the weak topology defined by the other; the concrete realization as a sub-vectorlattice in $M$ of the dual of any (order) closed sub-vector-lattice in $L$. Some of the results are to be found in Nakamura's paper [11]; he obtains them from the properties of $(L)$-spaces, while we prefer the more general development of Nakano.

In $\$ 5$ we characterize intrinsically the subspaces in $L$ of the form $\mathfrak{L}^{\mathfrak{l}}(\mu)$ and the subspaces in $M$ of the form $\mathscr{L}^{\infty}(\mu), \mu$ a Radon measure. In $\S 6$ we characterize the subsets of $L$ relatively compact under $w(L, M)$; and dual to this, in $\S 7$, we describe the Mackey topology $\tau(M, L)$.

In $\S 8$, we study $L$ in relation to $U, B o$, and $B a$ rather than to $M$, and show $L$ is the set of regular measures on these spaces. Some insight is obtained into the role of regularity.

Presented to the Society, November 30, 1957; received by the editors July 26, 1957 and, in revised form, July $8,1958$.

(1) The work on this paper was supported in part by National Science Foundation Grant NSF-G2988 and in part by a Faculty Research Grant from Wayne State University. 
The full Banach lattice dual $\Omega(M)$ of $M$ is examined (mildly) in $\S 9$. This is equivalent to studying the relations between Radon measures and various families of finitely additive measures.

2. Preliminaries. We assume a knowledge of [10]. A set $A$ in a vector lattice $E$ will be called order-bounded if there exist $b \in E, c \in E$ such that $b \leqq a \leqq c$ for all $a \in A$. $A$ will be called order-closed if an element $b$ of $E$ lies in $A$ whenever it is the infimum or supremum of some subset of $A$. Given $a \leqq b$ in $E$, the set $\{c \mid a \leqq c \leqq b\}$ will be denoted by $[a, b]$ and called an interval.

The letters $I$ and $J$ will always denote ideals. The notation $E=I \oplus J$ will mean that $E$ is the direct sum of $I$ and $J$. Given such a direct sum, $a_{I}$ will denote the component of the element $a$ in $I$ and $A_{I}$ will denote the projection $\left\{a_{I} \mid a \in A\right\}$, and similarly for $a_{J}$ and $A_{J}$. If $E=I \oplus J$ and $K$ is an ideal, then $K=(K \cap I) \oplus(K \cap J)(\cap$ denotes set-intersection).

A mapping of $E$ into another vector lattice will be called an isomorphism (into) if it is one-one, linear, and preserves the operations $\wedge$ and $\vee$. If it is also onto, then the last condition can be replaced by the weaker one that it preserves order.

We recall that $\Omega(E)$ (the vector lattice of bounded linear functionals on $E$ ) may be denoted by $\Omega$ when no confusion can result. If $A$ is a subset of $E$, then by the null-ideal of $A$ in $\Omega$, we will mean the set

$$
\{\phi \in \Omega|| \phi|| a \mid=0 \text { for all } a \in A\} .
$$

It is clearly an ideal, and is the largest ideal in $A^{\perp}$-in- $\Omega$ (the null-space of $A$ in $\Omega$ ). If $I$ is the ideal in $E$ generated by $A$, the null-ideal of $A$ in $\Omega$ is precisely $I^{\perp}$-in- $\Omega$; thus in particular, for an ideal, the null-space and null-ideal coincide. The null-ideal in $E$ of a subset of $\Omega$ is defined in the same way, and the same remarks hold.

The following two properties are elementary, and we omit their proofs.

(2.1) The null-ideal in $\Omega$ of a subset of $E$ is order-closed.

(2.2) If $E=I \oplus J$, then $\Omega=\left(I^{\perp}-i n-\Omega\right) \oplus\left(J^{\perp}-i n-\Omega\right)$.

A subset $A$ of $\Omega$ will be called separating on $E$ if $A^{\perp-i n-E}=0$; and similarly with $\Omega$ and $E$ interchanged. From (2.2) we obtain easily

(2.3) Let $\Omega$ be separating on $E$. Then if $E=I \oplus J, I$ and $J$ are $w(E, \Omega)$-closed. In particular, if $E$ is complete, every order-closed ideal in $E$ is $w(E, \Omega)$-closed.

REMARK. The above three theorems no longer necessarily hold, if we interchange $E$ and $\Omega$.

(2.4) Let $F$ be a sub-vector-lattice of $E$, and $\pi: \Omega \rightarrow \Omega(F)$ the (linear) mapping defined by $\pi \phi=\phi \mid F$ (the restriction of $\phi$ to $F$ ). Then

(1) $\pi \Omega$ is an ideal in $\Omega(F)$.

(2) $\pi\left(\Omega_{+}\right)=(\pi \Omega)_{+}$; in particular $\pi$ preserves order (however, it need not preserve $\wedge$ and $\vee$ ).

(3) If $F$ is separating on $\Omega, \pi$ is an isomorphism (into). 


\section{Proof.}

Lemma. If $\omega$ is a positive linear functional on $E$ and $\rho$ a positive linear functional on $F$ such that $\rho(a) \leqq \omega(a)$ for every $a \in F_{+}$, then $\rho$ can be extended to a positive linear functional on $E$ which is $\leqq \omega$.

If we define the sublinear functional $p(a)=\omega\left(a^{+}\right)$on $E$, it clearly dominates $\rho$ on $F$, and the lemma then follows from the Hahn-Banach theorem. We proceed to prove the theorem. That $\phi \geqq 0$ implies $\pi \phi \geqq 0$, and therefore $\pi$ preserves order, is trivial. Moreover,

(i) if $\phi \in \Omega$ and $0 \leqq \rho \leqq \pi \phi$ in $\Omega(F)$, then $\rho=\pi \psi$ for some $\psi \in \Omega_{+}$. To show this, we note that $\rho \leqq \pi \phi \leqq \pi|\phi|$ and thus we need only let $|\phi|$ be the $\omega$ of the lemma. (1) and (2) now follow directly from (i). If $F$ is separating on $\Omega, \pi$ is one-one and we oftain (3) (using (1)).

If $F$ in (2.4) is actually an ideal $I$, then $\Omega=I^{\perp} \oplus\left(I^{\perp}\right)^{\prime}$ (we write $I^{\perp}$ for $I^{\perp}$. in $\Omega$ ), and $\pi$ is one-one on $\left(I^{\perp}\right)^{\prime}$. This gives

(2.5) If in (2.4), $F$ is an ideal $I$, then $\pi$ maps $\left(I^{\perp}\right)^{\prime}$ isomorphically onto $\pi \Omega$.

Thus we can identify $\left(I^{\perp}\right)^{\prime}$ with an ideal in $\Omega(I)$. If moreover $E=I \oplus J$, then clearly $\left(I^{\perp}\right)^{\prime}=\Omega(I)$.

If $\Omega$ is separating on $E$, the definition $a(\phi)=\phi(a), a \in E, \phi \in \Omega$, gives a natural imbedding of $E$ in $\Omega(\Omega)$ as a linear subspace. In fact, we have more.

(2.6) If $\Omega$ is separating on $E$, then under its natural imbedding in $\Omega(\Omega)$, $E$ is a sub-vector-lattice: for every $a \in E, b \in E, a \bigvee b=a \bigvee b$-in- $\Omega(\Omega)$.

Proofs of this can be found in [3, p. 39, Problem 6] and [16; Theorem 7.9]. (This theorem makes unnecessary the proof we gave for the special case $[10,(5.1)]$.)

3. Continuity. As we stated in the introduction, this section is devoted to a self-contained exposition of the part of Nakano's theory of continuous linear functionals which we will need. Let $E$ be a vector lattice. The notation $\left\{a_{\alpha}\right\}$ will always indicate a net, that is, the index system $\{\alpha\}$ is a directed set under an order $<$. The notation $a_{\alpha} \uparrow a$ will mean that $a_{\alpha} \leqq a_{\beta}$ whenever $\alpha<\beta$, and $a=\mathrm{V}_{\alpha} a_{\alpha}$; and similarly for $a_{\alpha} \downarrow a$. These are special cases of convergence. We will say a net $\left\{a_{\alpha}\right\}$ converges to $a$-in notation $a_{\alpha} \rightarrow a$-if there exists $\left\{b_{\alpha}\right\}$ such that $b_{\alpha} \downarrow 0$ and $\left|a_{\alpha}-a\right| \leqq b_{\alpha}$ for all $\alpha$. If $E$ is complete, this is equivalent to the statement that $\left\{a_{\alpha}\right\}$ is order-bounded and $\lim \inf _{\alpha} a_{\alpha}=\lim \sup _{\alpha} a_{\alpha}=a$.

A subset will be called closed if it is closed under convergence. The closure $\bar{A}$ of any subset $A$ will be the intersection of the closed sets containing $A$. $A$ will be called dense in $B$, if $A \subset B$ and every element of $B$ is the limit of some net in $A$. Unlike the situation in topology, a set $A$ may not be dense in its closure, that is we cannot obtain $\bar{A}$ by simply adjoining to $A$ all the limits of nets of $A$.

REMARK 1. The above definitions illustrate our general policy on notation. Terms usually considered topological will be defined with respect to the above convergence. If we want to use such a term in the usual sense with respect to 
some topology $T$, we will modify it with $T$, e.g. $T$-closed, $T$-dense, etc.

REMARK 2. Since convergence preserves the operations $\wedge$ and $\vee$, it is easy to show, using Zorn's lemma, that the closure of a sub-vector-lattice is also a sub-vector-lattice. A closed sub-vector-lattice is order-closed. If $E$ is complete, every order-closed subset is closed.

REMARK 3. An ideal $I$ is dense in its closure $\bar{I}$. In fact, $\bar{I}_{+}$can be obtained from $I_{+}$by adjoining the suprema of all subsets of $I_{+}$. In particular, an ideal is closed if and only if it is order-closed.

An element $\phi$ of $\Omega$ will be called continuous if $\lim _{\alpha} \phi\left(a_{\alpha}\right)=\phi(\alpha)$ whenever $a_{\alpha} \rightarrow a$ (Nakano uses the term "universally continuous," Nakamura, the term "order-continuous"). An equivalent condition, more convenient to apply, is that $\lim _{\alpha}|\phi|\left(a_{\alpha}\right)=0$ whenever $a_{\alpha} \downarrow 0$. If $E$ is archimedean-hence, a fortiori if it is complete-we need not assume $\phi \in \Omega$ (only that is it linear), since the continuity condition then implies it is bounded.

(3.1) The continuous linear functionals on $E$ constitute a closed ideal in $\Omega$ We denote it by $\tilde{\Omega}(E)$, or simply $\tilde{\Omega}$.

Proof. That $\tilde{\Omega}$ is a linear subspace of $\Omega$ is trivial. That it is an ideal follows from the equivalent definition of continuity above. That it is, finally, closed, is shown by the

Lemma. Given $\phi_{\alpha} \uparrow \phi$ in $\Omega_{+}$, if the $\phi_{\alpha}$ 's are all continuous, then $\phi$ is also.

We remark first that for each $a \in E_{+}, \lim _{\alpha} \phi_{\alpha}(b)=\phi(b)$ uniformly on the interval $[-a, a]$. For, $\left|\phi(b)-\phi_{\alpha}(b)\right| \leqq\left(\phi-\phi_{\alpha}\right)|b| \leqq\left(\phi-\phi_{\alpha}\right)(a)$, which converges to zero independently of $b$. Now suppose $a_{\beta} \downarrow 0$; and we can assume there is a first member $a_{1}$. Since $0 \leqq a_{\beta} \leqq a_{1}$ for all $\beta$, it follows from the above remark that $\lim _{\alpha} \phi_{\alpha}\left(a_{\beta}\right)=\phi\left(a_{\beta}\right)$ uniformly in $\beta$. Since also $\lim _{\beta} \phi_{\alpha}\left(a_{\beta}\right)=0$ for each $\alpha$, standard function theory gives us that $\lim _{\beta} \phi\left(a_{\beta}\right)=0$. This proves the lemma and the theorem.

It is immediate from the above theorem that (2.1), (2.2), and (2.3) remain true if we replace $\Omega$ by $\tilde{\Omega}$. We show that now, moreover, the duals also hold (cf. the Remark after (2.3)).

(3.2) The null-ideal in $E$ of a subet $A$ of $\tilde{\Omega}$ is closed.

Proof. If $A$ consists of a single element, the statement follows from the definition of continuity. But the null-ideal of a general subset $A$ is the intersection of the null-ideals of its elements, which completes the proof.

(3.3) If $E$ is complete, every closed ideal I in $\tilde{\Omega}$ is $w(\tilde{\Omega}, E)$-closed.

Proof. Since $\Omega$ is complete, $\tilde{\Omega}$ is also. Hence $\tilde{\Omega}=I \oplus I^{\prime}$ (we write $I^{\prime}$ for $I^{\prime}$-in- $\left.\widetilde{\Omega}\right)$. It is sufficient to show that if $\phi \in I^{\prime}, \phi \neq 0$, then $\phi$ is not a $w(\tilde{\Omega}, E)$ limit-point of $I$. The null-ideal $J$ of $\phi$ in $E$ is closed (3.2), hence $E=J \oplus J^{\prime}$. This gives in turn, $\widetilde{\Omega}=J^{\perp} \oplus J^{\perp \perp}$ (writing $J^{\perp}$ for $J^{\perp}$-in- $\widetilde{\Omega}$ and $J^{\prime \perp}$ for $\left(J^{\prime}\right)^{\perp}$-in- $\widetilde{\Omega}$ ). Since $\phi \in J^{\perp}$ and $J^{\perp}$ is $w(\tilde{\Omega}, E)$-closed, we can complete the proof by showing $I \subset J^{\perp}$, or equivalently that $I \cap J^{\perp}=0$.

Consider any $\psi \in I, \psi>0$. We have to show $\psi(a) \neq 0$ for some $a \in J$. Now 
$\psi \wedge|\phi|=0$, hence [3, p. 36, Proposition 3] for every $\epsilon>0$ and $b \in E_{+}$, there exists $c \in E$ such that $0 \leqq c \leqq b,|\phi|(c)+\psi(b-c) \leqq \epsilon$. It follows readily that there exists a sequence $c_{1} \geqq c_{2} \geqq \cdots \geqq 0$ in $E$ satisfying $|\phi|\left(c_{n}\right) \leqq 1 / 2^{n}$ and $\psi\left(c_{n}\right) \geqq \lambda>0$ for all $n$. Taking $a=\Lambda_{n} c_{n}$, we have $|\phi|(a)=0$ and (since $\psi$ is continuous) $\psi(a)>0$.

(3.4) Let $E$ be complete and $\tilde{\Omega}$ separating on $E$. If $\Omega=I \oplus J$, then $E=\left(I^{\perp}\right.$-in-E) $\oplus\left(J^{\perp}\right.$-in- $\left.E\right)$.

Proof. We omit all designations "in $E$ " and "in $\tilde{\Omega}$, " since the context will be clear. We recall also that (2.1), (2.2), and (2.3) hold with $\Omega$ replaced by $\tilde{\Omega}$. From (3.2), $I^{\perp}$ is closed, hence $E=I^{\perp} \oplus\left(I^{\perp}\right)^{\prime}$. We show $\left(I^{\perp}\right)^{\prime}=J^{\perp}$. On the one hand, since $\tilde{\Omega}$ is separating on $E, I^{\perp} \cap J^{\perp}=0$, hence $J^{\perp} \subset\left(I^{\perp}\right)^{\prime}$. On the other hand, from (2.2) and (3.3), $\tilde{\Omega}=I^{\perp \perp} \oplus\left(I^{\perp}\right)^{\prime \perp}=I \oplus\left(I^{\perp}\right)^{\prime \perp}$, whence $\left(I^{\perp}\right)^{\prime \perp}=J$ and therefore $\left(I^{\perp}\right)^{\prime} \subset J^{\perp}$.

REMARK 1. It is not hard to show that (3.4) still holds if $\tilde{\Omega}$ is replaced by any ideal in it which is also separating on $E$.

REMARK 2. Given $\phi \in \tilde{\Omega}$, let $I$ be the closed ideal generated by $\phi^{+}, J$ that generated by $\phi^{-}$, and $K$ the (closed) ideal of elements disjoint from $\phi$. Then $\tilde{\Omega}=I \oplus J \oplus K$, and the above theorem gives a decomposition $E=I_{1} \oplus J_{1} \oplus K_{1}$ with the property that $\phi(a)>0$ for all $a>0$ in $I_{1}, \phi(a)<0$ for all $a>0$ in $J_{1}$, and $\phi(a)=0$ for all $a \in K[13$, Theorem 1].

(3.5) Let $F$ be a closed sub-vector-lattice of $E$ and $\pi$ the mapping of (2.4). Then

(a) $\pi$ carries $\tilde{\Omega}$ into $\tilde{\Omega}(F)$ and (1), (2), (3) of (2.4) hold with $\Omega, \Omega(F)$ replaced by $\tilde{\Omega}, \widetilde{\Omega}(F)$.

(b) If $E$ is complete and $\tilde{\Omega}$ is separating on $E$, then $\pi \tilde{\Omega}$ is dense in $\tilde{\Omega}(F)$.

Moreover, if $F$ is an ideal, these statements hold with no assumption of closedness on it.

Proof. Since $F$ is closed, $a_{\alpha} \downarrow 0$ in $F$ implies $a_{\alpha} \downarrow 0$ in $E$. Hence if $\phi$ is continuous on $E, \phi \mid F$ is continuous on $F$. The rest of (a) is easily verified. To prove (b), we note first that $F$, being closed, is complete. It follows from (3.3) that the closure of $\pi \widetilde{\Omega}$ in $\widetilde{\Omega}(F)$ is a $w(\widetilde{\Omega}(F), F)$-closed ideal. But $\pi \tilde{\Omega}$ is separating on $F$, hence $w(\tilde{\Omega}(F), F)$-dense in $\widetilde{\Omega}(F)$, and therefore its closure is identical with $\tilde{\Omega}(F)$. The above argument holds for an arbitrary ideal in $E$, closed or not (since every ideal in $E$ is complete), which establishes the last statement.

(3.6) Let $E$ be complete and $\tilde{\Omega}$ separating on $E$. If $F$ is a sub-vector-lattice of $E$, the following statements are equivalent:

$1^{\circ} . \bar{F}=E$.

$2^{\circ} . F$ is $w(E, \tilde{\Omega})$-dense in $E$.

$3^{\circ}$. $F$ is separating on $\tilde{\Omega}$.

Proof. From the definition of $\tilde{\Omega}$, if a net in $E$ converges, it $w(E, \tilde{\Omega})$-converges. Thus $1^{\circ}$ implies $2^{\circ}$. That $2^{\circ}$ and $3^{\circ}$ are equivalent is a standard property of vector spaces. Now assume $3^{\circ}$ (hence $2^{\circ}$ ) and consider $\bar{F}$. As we have remarked, it is a (closed) sub-vector-lattice. We show first, 
(i) if $E=I \oplus J, \bar{F}=(I \cap \bar{F}) \oplus(J \cap \bar{F})$.

From (2.2) $\tilde{\Omega}=I^{\perp} \oplus J^{\perp}$ (we omit “in $\tilde{\Omega}$ "). Now from (3.5) and (2.4), $\tilde{\Omega}$ can be considered an ideal in $\tilde{\Omega}(F)$, hence (Remark 1 following $(3.4)), \bar{F}=\left(\left(I^{\perp}\right)^{\perp}\right.$-in- $\left.\bar{F}\right)$ $\oplus\left(\left(J^{\perp}\right)^{\perp}\right.$-in- $\left.\bar{F}\right)$. Since $I=\left(I^{\perp}\right)^{\perp}$-in- $E$ and $J=\left(J^{\perp}\right)^{\perp}$-in- $E$ (3.3), this can be written $\bar{F}=(I \cap \bar{F}) \oplus(J \cap \bar{F})$. We show next

(ii) $\bar{F}$ is an ideal in $E$.

Since $\bar{F}$ is a sub-vector-lattice, we need only show that $0 \leqq b \leqq a, a \in \bar{F}$ implies $b \in \bar{F}$. Suppose we have such an $a$ and $b$, but $b \in \bar{F}$. We can assume there is no element of $\overline{\boldsymbol{F}}$ properly between 0 and $b$. For, if there is, then there is a largest one $c$; and we need only replace $b$ by $b-c$. Now $(1 / n) a \downarrow 0$, hence $b-(1 / n) a \uparrow b$, hence in turn $(b-(1 / n) a)^{+} \uparrow b$. Since $b>0$, we must have $(b-(1 / n) a)^{+}>0$ for some $n$. Thus, denoting this $1 / n$ by $\lambda$, we have that $(b-\lambda a)^{+}>0$ for some $\lambda>0$.

Let $I$ be the closed ideal in $E$ generated by $(b-\lambda a)^{+}$. Then $E=I \oplus I^{\prime}$, hence from (i), $\bar{F}=(I \cap \bar{F}) \oplus\left(I^{\prime} \cap \bar{F}\right)$. Writing $a=a_{I}+a_{I^{\prime}}=a_{I \cap \bar{F}}+a_{I^{\prime} \cap \bar{F}}$, the uniqueness of components gives us $a_{I}=a_{I \cap} \bar{F} \in \bar{F}$. We show $0<\lambda a_{I}<b$, which will contradict our assumption on $b . b_{I}-\lambda a_{I}=(b-\lambda a)_{I}=(b-\lambda a)^{+}>0$, whence $b_{I}>\lambda a_{I}$ and therefore $b \geqq b_{I}>\lambda a_{I}$. That $\lambda a_{I}>0$ follows from the fact that $a \geqq(b-\lambda a)^{+}>0$, hence $a_{I} \geqq\left[(b-\lambda a)^{+}\right]_{I}=(b-\lambda a)^{+}>0$, hence $\lambda a_{I}>0$.

Thus (ii) is established, and $\bar{F}$ is a closed ideal in $E$. From (2.3) (with $\tilde{\Omega}$ replacing $\Omega), \bar{F}$ is $w(E, \tilde{\Omega})$-closed. Since it is $w(E, \tilde{\Omega})$-dense, it must coincide with $E$. This completes the proof.

As we know (2.6), if $\Omega$ is separating on $E$, then under its natural imbedding in $\Omega(\Omega), E$ is a sub-vector-lattice. Moreover, each element of $E$ is clearly continuous on $\Omega[10,(2.2)]$, and thus $E \subset \widetilde{\Omega}(\Omega)$. We have in fact,

(3.7) Let $\Omega$ be separating on $E$. Then under the natural imbedding of $E$ in $\Omega(\Omega)$, the closed ideal generated by $E$ is $\widetilde{\Omega}(\Omega)$.

Proof. Let $I$ be this closed ideal. Since $\tilde{\Omega}(\Omega)$ is a closed ideal, we have $I \subset \tilde{\Omega}(\Omega)$. From (3.3), it is $w(\tilde{\Omega}(\Omega), \Omega)$-closed in $\tilde{\Omega}(\Omega)$. But $E$ is $w(\tilde{\Omega}(\Omega), \Omega)$-dense in $\widetilde{\Omega}(\Omega)$, hence $I$ is also. It follows $I=\widetilde{\Omega}(\Omega)$.

REMARK. We will give a considerably stronger theorem in (3.10) below.

Suppose now that $\widetilde{\Omega}$ is separating on $E$. Then the above discussion can all be carried out with $\tilde{\Omega}$ replacing $\Omega$ : the definition $a(\phi)=\phi(a)$ gives a natural imbedding of $E$ in $\Omega(\tilde{\Omega})$; under this imbedding, $E$ is a sub-vector-lattice; and the closed ideal generated by $E$ is $\tilde{\Omega}(\tilde{\Omega})$. However, we can now say more.

(3.8) Let $E$ be complete. If $\tilde{\Omega}$ is separating on $E$, then under the natural imbedding of $E$ in $\tilde{\Omega}(\tilde{\Omega}), E$ is an ideal (which is dense in $\tilde{\Omega}(\tilde{\Omega})$ ).

Proof. Since $E \subset \tilde{\Omega}(\tilde{\Omega})$, we can confine our attention to the latter space rather than $\Omega(\tilde{\Omega})$. If we let $\tilde{\Omega}(\tilde{\Omega}), \tilde{\Omega}, E$ play the roles of $E, \tilde{\Omega}, \bar{F}$ respectively in (3.6), some minor modification of the proof of (ii) there gives us the desired result.

Corollary. If $E$ is complete, the intervals of $E$ are $w(E, \tilde{\Omega})$-compact.

Proof. Consider $a \in E, b \in E, a<b$. From the above theorem, the interval 
$[a, b]$ in $\Omega(\tilde{\Omega})$ is contained in $E$, hence is also the interval $[a, b]$ in $E$. Since intervals in $\Omega(\tilde{\Omega})$ are $w(\Omega(\widetilde{\Omega}), \tilde{\Omega})$-compact [10, (10.3)], we are through.

We will say $E$ is reflexive with respect to continuity if $E=\widetilde{\Omega}(\widetilde{\Omega})$, that is, if its natural imbedding in $\tilde{\Omega}(\tilde{\Omega})$ is onto. (This definition implies of course that $\tilde{\Omega}$ is separating on $E$.) Although other kinds of reflexivity could be considered -for example, $E=\Omega(\Omega)$-the above is the only one we will need in this paper. While a vector lattice is not in general reflexive with respect to continuity, the following theorem gives a large class of vector lattices which are.

(3.9) $\Omega$ is reflexive with respect to continuity. More generally, this is true for every closed sub-vector-lattice of $\Omega$, hence in particular for $\widetilde{\Omega}$.

Proof. Since $E \subset \tilde{\Omega}(\Omega)$, the latter is separating on $\Omega$. It follows from (3.8) that $\Omega$ is a dense ideal in $\widetilde{\Omega}(\widetilde{\Omega}(\Omega))$. This means that for each positive element $\psi$ of $\tilde{\Omega}(\tilde{\Omega}(\Omega))$, there is some net $\left\{\phi_{\alpha}\right\}$ in $\Omega$ such that $\phi_{\alpha} \uparrow \psi$. We show $\psi \in \Omega$. $\phi_{\alpha}(f) \leqq \psi(f)$ for every $\alpha$ and every $f \geqq 0$ in $\widetilde{\Omega}(\Omega)$, hence in particular for every such $f$ in $E$. Applying $[10,(11.6)]$, there exists $\phi=\mathrm{V}_{\alpha} \phi_{\alpha}$-in- $\Omega$. Then $0 \leqq \psi \leqq \phi$, and since $\Omega$ is an ideal in $\tilde{\Omega}(\tilde{\Omega}(\Omega)), \psi \in \Omega$.

Now suppose $F$ is a closed sub-vector-lattice of $\Omega$. Then $F$ also satisfies the conditions of (3.8), that is, it is complete and $\tilde{\Omega}(F)$ is separating on $F$ (since $\tilde{\Omega}(F) \supset \pi \tilde{\Omega}(\Omega)(3.5)$ ). It follows $F$ is a dense ideal in $\tilde{\Omega}(\tilde{\Omega}(F))$. The argument used above for $\Omega$ now gives us that $F=\tilde{\Omega}(\tilde{\Omega}(F))$.

We can now state the promised strengthening of (3.7).

(3.10) Let $\Omega$ be separating on $E$. Then under the natural imbedding of $E$ in $\Omega(\Omega), \bar{E}=\tilde{\Omega}(\Omega)$.

Proof. Under the imbedding, $E$ is a sub-vector-lattice of $\widetilde{\Omega}(\Omega)$. From (3.9), $\Omega=\tilde{\Omega}(\widetilde{\Omega}(\Omega)$ ). Letting $\tilde{\Omega}(\Omega)$ be the $E$ of (3.6), we obtain the desired conclusion.

4. Applications to $L$ and $M$. As we know, the dual $M=\Omega(L)$ of $L$ is also its Banach space dual [10, (3.8)]. Now $L$, being an $(L)$-space, has the property that $\lim _{\alpha}\left\|\mu_{\alpha}\right\|=0$ whenever $\mu_{\alpha} \downarrow 0$. It follows that every element of $M$ is continuous:

(4.1) $M=\Omega(L)=\tilde{\Omega}(L)$.

This gives, via (3.9),

(4.2) $L=\widetilde{\Omega}(M)$,

and, via (3.10),

(4.3) The closure of $C$ in $M$ is $M$ itself.

REMARK 1. In [10] we showed that the set of elements of $M$ which are limits of nets of $C$ is the sub-vector-lattice $U$. $U$ is in general not all of $M$, hence from the above, is not closed (although it is closed under sequential convergence). By adjoining limits of nets of $U$, we could obtain a larger subvector-lattice of $M$; and this process could be continued, in the same way as the standard process for obtaining the Baire classes, until all of $M$ is exhausted (the resulting well-ordered family would presumably go beyond the first uncountable ordinal).

REMARK 2. If a net in $M$ converges, it $|w|(M, L)$-converges [10, (11.7)]; hence (4.3) gives us immediately that $C$ is $|w|(M, L)$-dense in $M$. We thus 
obtain the result $[10,(11.9)]$ by a completely different proof than the one used there.

From (3.2),

(4.4) The null-ideal in $L$ or $M$ of a subset of the other is closed.

From (3.4),

(4.5) If $L$ or $M$ is the direct sum of two ideals, the other is the direct sum of their null-spaces.

It is also easily shown that

(4.6) The correspondence $I \leftrightarrow\left(I^{\perp}\right)^{\prime}$ is an isomorphism of the lattice of closed ideals in $L$ or $M$ with that of closed ideals in the other.

We turn now to closed sub-vector-lattices of $L$ and $M$. The results are still essentially due to Nakano. From (3.9) we have

(4.7) Every closed sub-vector-lattice of $L$ or $M$ is reflexive with respect to continuity.

For closed ideals, this duality can even be obtained by concrete realizations. If $I$ is a closed ideal in $L,\left(I^{\perp}\right)^{\prime}$-in- $M$ can be identified with $\Omega(I)=\widetilde{\Omega}(I)$; if it is a closed ideal in $M,\left(I^{\perp}\right)^{\prime}$-in- $L$ can be identified with $\tilde{\Omega}(I)$. We want to know to what extent such realizations are obtainable for general closed subvector-lattices.

We will need

(4.8) The mapping $\pi$ of (2.4) is continuous, that is, preserves convergence.

Proof. We have to show that $\phi_{\alpha} \rightarrow \phi$ in $\Omega$ implies $\pi \phi_{\alpha} \rightarrow \pi \phi$ in $\Omega(F)$. The former means there exist $\psi_{\alpha} \uparrow \phi$ and $\omega_{\alpha} \downarrow \phi$ such that $\psi_{\alpha} \leqq \phi_{\alpha} \leqq \omega_{\alpha}$ for all $\alpha$. Since $\pi$ preserves order, we have $\pi \psi_{\alpha} \leqq \pi \psi_{\beta}$ whenever $\psi_{\alpha} \leqq \psi_{\beta}, \pi \omega_{\alpha} \geqq \pi \omega_{\beta}$ whenever $\omega_{\alpha} \geqq \omega_{\beta}$, and $\pi \psi_{\alpha} \leqq \pi \phi_{\alpha} \leqq \pi \omega_{\alpha}$ for all $\alpha$. Hence it is enough to show that $\pi \phi=\mathrm{V}_{\alpha} \pi \psi_{\alpha}=\Lambda_{\alpha} \pi \omega_{\alpha}$, or equivalently, that for every $a \in F_{+}, \phi(a)=\sup _{\alpha} \psi_{\alpha}(a)$ $=\inf _{\alpha} \omega_{\alpha}(a)$. Since this is true on $E_{+}$, it is a fortiori true on $F_{+}$.

Now in general $\pi \Omega$ is not all of $\Omega(F)$. However,

(4.9) If $F$ is a closed sub-vector-lattice of $L$, then $\pi M=\Omega(F)=\tilde{\Omega}(F)$.

Proof. $F$ is clearly a norm-closed linear subspace of $L$, and in fact an $(L)$-space. It follows from Banach space theory that $\pi M=M / F^{\perp}=$ the Banach space dual of $F$; hence from Banach lattice theory that $\pi M=\Omega(F)$; finally from the $(L)$-space property (cf. (4.1)), that $\Omega(F)=\widetilde{\Omega}(F)$.

We can also obtain our concrete realization of $\Omega(F)$ :

(4.10) If $F$ is a closed sub-vector-lattice of $L$, there is a uniquely determined closed sub-vector-lattice $E$ of $M$ such that $\pi$ maps $E$ isomorphically onto $\Omega(F)$.

Proof. We will define a mapping $p: \Omega(F) \rightarrow M$ which is an isomorphism into and whose inverse (on the image) is $\pi$. Let $I$ be the null-ideal of $F$ in $M$, and $J=I^{\prime}$. Then $\pi J=\Omega(F)$, and $\pi$ considered only on $J$ has all the properties. of (3.5) and (4.8). Moreover, clearly,

(i) for $f \in J_{+}, \pi f=0$ implies $f=0$.

This gives in turn,

(ii) for $f \in J_{+}, g \in J_{+}, \pi f \wedge \pi g=0$ implies $f \wedge g=0$. 
For, since $\pi$ preserves order, $0 \leqq \pi(f \wedge g) \leqq \pi f \wedge \pi g=0$, hence $\pi(f \wedge g)=0$, and the required conclusion follows from (i).

We remark that $1_{J}$ is a strong order unit for $J, \pi 1$ is one for $\Omega(F)$, and $\pi 1_{J}=\pi 1$. We show now that for each component $d$ of $\pi 1$ (that is, $d \wedge(\pi 1-d)$ $=0)$, there is one and only one component $e$ of $1_{J}$ such that $\pi e=d$; we will then set this $e=p d$. Now $0 \leqq d \leqq \pi 1$, hence from the lemma of (2.4), there exists $e$ satisfying $0 \leqq e \leqq 1_{J}$ and $\pi e=d$. Since $\pi\left(1_{J}-e\right)=\pi 1-d$, (ii) gives us $e \wedge\left(1_{J}-e\right)=0$, and thus $e$ is a component of $1_{J}$. If $e_{1}$ is any component of $1_{J}$ such that $\pi e_{1}=d$, then by (ii) again, $e \wedge\left(1_{J}-e_{1}\right)=0$, hence $e \leqq e_{1}$ and (i) then gives $e=e_{1}$. Thus $e$ is unique and will be our $p d$.

If $d_{1} \leqq d_{2}$, then the above argument-with $d_{2}$ replacing $\pi 1$-gives us that $p d_{1} \leqq p d_{2}$. We have even more:

(iii) For any two components $d_{1}, d_{2}$ of $\pi 1, p\left(d_{1} \wedge d_{2}\right)=p d_{1} \wedge p d_{2}$. Since $d_{1} \wedge d_{2}$ is $\leqq$ both $d_{1}$ and $d_{2}, p\left(d_{1} \wedge d_{2}\right)$ is $\leqq$ both $p d_{1}$ and $p d_{2}$; hence $p\left(d_{1} \wedge d_{2}\right) \leqq p d_{1} \wedge p d_{2}$, and therefore $d_{1} \wedge d_{2} \leqq \pi\left(p d_{1} \wedge p d_{2}\right)$. On the other hand, since $p d_{1} \wedge p d_{2}$ is $\leqq$ both $p d_{1}$ and $p d_{2}, \pi\left(p d_{1} \wedge p d_{2}\right) \leqq d_{1} \wedge d_{2}$. We thus have $d_{1} \wedge d_{2}=\pi\left(p d_{1} \wedge p d_{2}\right)$, hence from uniqueness, $p\left(d_{1} \wedge d_{2}\right)=p d_{1} \wedge p d_{2}$.

Let us denote by $[d]$ the linear subspace generated by the $d$ 's and by $[p d]$, that generated by the $p d$ 's. It follows easily from (iii) that $[d]$ and $[p d]$ are sub-vector-lattices and that the extension of $p$ to $[d]$ by linearity gives an isomorphism onto [pd] with inverse $\pi$.

Now from the Freudenthal integral representation $[2$, p. 231], for each $s \in \Omega(F)$, there is a sequence $\left\{s_{n}\right\} \subset[d]$ such that $s_{n} \uparrow s$. Since the set $\left\{s_{n}\right\}$ is bounded above by some multiple of $\pi 1,\left\{p s_{n}\right\}$ is also bounded above (by the same multiple of $1_{J}$ ), hence $p s_{n} \uparrow f$ for some $f \in J$. We set $p s=f$. Since $\pi$ preserves convergence, $\pi f=s$. Moreover, $f$ is defined uniquely. For suppose we also have $t_{n} \uparrow s,\left\{t_{n}\right\} \subset[d]$. Let $p t_{n} \uparrow g$. Then $p s_{n} \wedge p t_{n} \uparrow f \wedge g$, hence $s_{n} \wedge t_{n}=\pi p s_{n} \wedge \pi p t_{n}=\pi\left(p s_{n} \wedge p t_{n}\right) \uparrow \pi(f \wedge g)$. But $s_{n} \wedge t_{n} \uparrow s$; therefore $\pi(f \wedge g)$ $=s$. It follows from (i) that $f=f \wedge g=g$.

We thus have extended $p$ to all of $\Omega(F)$, so that $p^{-1}=\pi$ and

(iv) if $s_{n} \uparrow s$ in $\Omega(F),\left\{s_{n}\right\} \subset[d]$, then $p s_{n} \uparrow p s$.

We show that for any $s \in \Omega(F), p s^{+}=(p s)^{+}$, whence it will follow $p$ preserves the operations $\wedge, \vee$. Let $s_{n} \uparrow s,\left\{s_{n}\right\} \subset[d]$. Then also $s_{n}^{+} \uparrow s^{+}$, hence from (iv), $p s_{n} \uparrow p s$ and $p\left(s_{n}^{+}\right) \uparrow p\left(s^{+}\right)$. The first of these gives $\left(p s_{n}\right)^{+} \uparrow(p s)^{+}$. Since $p\left(s_{n}^{+}\right)=\left(p s_{n}\right)^{+}$, it follows $p\left(s^{+}\right)=(p s)^{+}$.

This last result gives us that $p \Omega(F)$ is a sub-vector-lattice $E$ of $J$, and that $p: \Omega(F) \rightarrow E$ is an isomorphism. It remains only to show that $E$ is order-closed (Remark 2 at the beginning of $\S 3$ ). Suppose $A \subset E$ and $f=\mathrm{V} A$. Let $s=\mathrm{V}(\pi A)$. Then $p s \geqq A$, hence $A \leqq f \leqq p s$, hence $\pi A \leqq \pi f \leqq s=\mathrm{V}(\pi A)$, hence $\pi f=s$. Applying (i), we obtain $f=p s \in E$. This completes the proof of (4.10).

Now let us consider the case of a closed sub-vector-lattice $F$ of $M$. Can we obtain analogues of (4.9) and (4.10) for such an $F$ ? The argument used in (4.9) would give us that $\pi \Omega(M)=\Omega(F)$. However $L=\widetilde{\Omega}(M)$, which is only a 
part of $\Omega(M)$, and we do not know whether $\pi L$ is all of $\tilde{\Omega}(F)$. To partially make up for this, we have a strengthening of (4.7) [12, Theorem 25.2].

(4.11) Let $F$ be a closed sub-vector-lattice of $M$ and $\pi: L \rightarrow \widetilde{\Omega}(F)$ defined by $\pi \mu=\mu \mid F$. Then $F=\tilde{\Omega}(\pi L)$.

Proof. Since $\pi L$ is a dense ideal in $\tilde{\Omega}(F)$ (3.5), it follows from (4.7) and the last statement in (3.5) that $F=\tilde{\Omega}(\tilde{\Omega}(F))$ is a dense ideal in $\tilde{\Omega}(\pi L)$. The same argument we used (twice) in (3.9) then gives us that $F=\tilde{\Omega}(\pi L)$.

Now what about an analogue of (4.10), at least for $\pi L$ if not for $\tilde{\Omega}(F)$ ? The procedure in (4.10) depended in an essential way on the existence of a strong unit, hence cannot be applied here as such. We content ourselves with stating the following realization theorem [12, Theorem 23.3], which we will need in (4.13).

(4.12) Let $F$ be a closed sub-vector-lattice of $M$. For each $\phi \in \pi L$, there is a sub-vector-lattice $E$ in $L$ such that

(a) $\pi$ maps $E$ isomorphically onto the ideal I generated by $\phi$, and

(b) every interval of $E$ is closed in $L$.

We prove, finally [12, Theorem 28.6],

(4.13) $A$ closed sub-vector-lattice $F$ of $L$ or $M$ is closed under the weak topology defined by the other.

Proof. If $F \subset L$ there is nothing to prove, since $F$ is also norm-closed and $M$ is the norm-dual of $L$. Suppose $F \subset M$. From the isomorphism of (4.12), we have

Lemma. If $\rho_{\alpha} \downarrow 0$ in $\pi L$, there exists $\mu_{\alpha} \downarrow 0$ in $L$ such that $\pi \mu_{\alpha}=\rho_{\alpha}$ for all $\alpha$.

Now to prove $F$ is $w(M, L)$-closed in $M$, we show $F^{\perp \perp}=F$. Consider $f \in F^{\perp \perp}$. $f$, having value zero on $F^{\perp}$, defines a linear functional $\phi$ on $\pi L$. Since $f$ is continuous on $L$, it follows from the lemma that $\phi$ is continuous on $\pi L: \phi \in \tilde{\Omega}(\pi L)$. Applying (4.11), $\phi$ coincides on $\pi L$ with an element $g$ of $F$. But then $f$ coincides with $g$ on all of $L$. It follows $f=g \in F$.

5. Principal closed ideals in $L$. Given an element $a$ of a vector lattice $E$, we will call the ideal (the closed ideal) generated by $a$ a principal ideal (a principal closed ideal). In $M$ every closed ideal $I$ is a principal ideal, being generated by $1_{I}$. In $L$ this is not the case.

Given $\mu \in L$, we will denote by $L_{\mu}$ the closed ideal generated by $\mu$, by $\nu_{\mu}$ the component in $L_{\mu}$ of an element $\nu$ of $L$, and by $A_{\mu}$ the projection $\left\{\nu_{\mu} \mid \nu \in A\right\}$ in $L_{\mu}$ of a subset $A$ of $L$. The dual closed ideal $\left(L_{\mu}^{\perp}\right)^{\prime}$-in- $M$ will be denoted by $M_{\mu}$; and for $f \in M, A \subset M, f_{\mu}$ and $A_{\mu}$ will be their component and projection, respectively, in $M_{\mu} . L_{\mu}$ is the set of measures which are absolutely continuous with respect to $\mu$, hence can be identified with $\mathscr{L}^{1}(\mu)$ (actually, we should say "absolutely continuous with respect to $|\mu|$ " and write $\mathscr{L}^{1}(|\mu|)$, but we will retain the above abuse of language). Since $M_{\mu}=\Omega\left(L_{\mu}\right)$, it can then be identified with $\mathscr{L}^{\infty}(\mu)$. Thus the principal closed ideals in $L$ and their dual ideals in $M$ are well-known objects, and a detailed study of them here 
is unnecessary. In the present section we give a few of their properties which we will need explicitly or which are related to $L$ and $M$ in the large.

Given $\mu \in L,\|\|_{\mu}$ will denote the semi-norm on $M$ defined by $\|f\|_{\mu}=|f||\mu|$. On $M_{\mu},\|\|_{\mu}$ becomes a norm. The set of components of 1 in $M$ will be denoted by $\varepsilon$. It is clear that for any $\mu \in L, \varepsilon_{\mu}=\varepsilon \cap M_{\mu}$. The usual definition of absolute continuity is as follows; $\nu$ is called absolutely continuous with respect to $\mu$ if $\nu$ is \|\|$_{\mu}$-continuous at 0 on $\varepsilon$. This definition of course comes from the standard development of integration theory via sets. In our present vector space approach, a more intuitive form is the one given in $[10,(12.1)]$, that $\nu$ is uniformly \|\|$_{\mu}$-continuous on $\Sigma(M)$ (the unit ball $[-1,1]$ of $M$ ). (In this connection, cf. (10.2) in the Appendix.)

If one (hence both) of these statements hold for $\nu$, then it holds also for $|\nu|$. We will need the following quantitative formulation of this last fact. Given $\epsilon>0$, there exists, by the definition of absolute continuity, a $\delta>0$ such that $|\nu(e)| \leqq \epsilon$ whenever $\|e\|_{\mu} \leqq \delta, e \in \mathcal{E}$; then for every such $e,|\nu|(e) \leqq 2 \epsilon$. To see this, write $M=I \oplus J$ such that $\nu \mid I \geqq 0$ and $\nu \mid J \leqq 0$ (cf. Remark 2 after (3.4)). Then $|\nu|(e)=|\nu|\left(e_{I}\right)+|\nu|\left(e_{J}\right)=\left|\nu\left(e_{I}\right)\right|+\left|\nu\left(e_{J}\right)\right| \leqq 2 \epsilon$.

As with any Banach lattice, a countable set of elements $\left\{\mu_{n}\right\}$ in $L$ is always contained in a principal ideal (e.g. the ideal generated by $\mu=\sum_{1}^{\infty}\left|\mu_{n}\right| / \lambda_{n}$, where $\left.\lambda_{n}=2^{n}\left\|\mu_{n}\right\|\right)$. Hence the union of a countable number of principal closed ideals is always contained in a principal closed ideal. The following characterization of principal closed ideals in $L$ is easily proved [18, Theorem 3].

(5.1) $A$ closed ideal in $L$ is an $L_{\mu}$ for some $\mu \in L$ if and only if every set of mutually disjoint nonzero elements is countable.

Now a natural problem is to characterize the $M_{\mu}$ 's among all the closed ideals in $M$, or equivalently, to characterize the components of 1 of the form $1_{\mu}$. In this direction, we have the following (cf. [14, Theorem 6.1]):

(5.2) If $I$ is a closed ideal in $M$, the following statements are equivalent:

1. $I=M \mu$ for some $\mu \in L$.

2. If $f_{\alpha} \downarrow 0$ in I, there exists a sequence of $\alpha^{\prime} s, \alpha_{1}, \alpha_{2}, \cdots$, such that $f_{\alpha_{n}} \downarrow 0$.

3 . Every set of mutually disjoint nonzero elements of $I$ is countable.

Proof. Suppose $I=M_{\mu}$ for some $\mu \in L$, and let $f_{\alpha} \downarrow 0$ in $M_{\mu}$. Then $\lim _{\alpha}\left\|f_{\alpha}\right\|_{\mu}$ $=0[10,(11.4)]$, and we can obtain the required subsequence. Thus $1^{\circ}$ implies $2^{\circ}$. Now assume $2^{\circ}$ and let $A$ be a set of mutually disjoint nonzero elements of $I$. We can assume the elements of $A$ are all $>0$; also that $A$ is orderbounded, since each $f \in A$ can be replaced by $f \wedge 1$. We now take for an index system $\{\alpha\}$ the set of finite subsets of $A$, ordered by inclusion; and for each $\alpha$, we set $g_{\alpha}=\bigvee_{f \in A, f \notin \alpha} f$. Then $g_{\alpha} \downarrow 0$. To show this, since clearly $g_{\alpha} \geqq g_{\beta}$ whenever $\alpha<\beta$, we need only show that $\Lambda_{\alpha} g_{\alpha}=0$. Let $g=\Lambda_{\alpha} g_{\alpha}$. For each $f \in A$, if we take for $\alpha$ the single element $f$, then $g \wedge f \leqq g_{\alpha} \wedge f=0$. It follows $g \wedge\left(\bigvee_{f \in A} f\right)$ $=0$. Since $g \leqq \bigvee_{f \in A} f$, this gives $g=0$. Now applying $2^{\circ}$, we have $\Lambda_{n} g_{\alpha_{n}}=0$ for some sequence $\left\{\alpha_{n}\right\}$. Let $B=\left\{f \mid f \in \alpha_{n}\right.$ for some $\left.n\right\}$. Then for $f \in A, f \in B$ 
we have $f \leqq g_{\alpha_{n}}$ for all $n$, hence $f=0$. Thus $A=B$, and $A$ is therefore countable.

Now assume $3^{\circ}$, and let $J=\left(I^{\perp}\right)^{\prime}$-in- $L$. We show $J=L_{\mu}$ for some $\mu$, which will prove $1^{\circ}$. It is enough (5.1) to show that every set $A$ of mutually disjoint nonzero elements of $J$ is countable. For each $\nu \in A, 1$, is in $I$, and since the $\nu$ 's are mutually disjoint, the $1{ }_{\nu}$ 's are also. Applying $3^{\circ}$, we have that $A$ is countable. This completes the proof of the theorem.

Corollary. A component $e$ of 1 is a $\mathbf{1}_{\mu}$ for some $\mu \in L$ if and only if e cannot have an uncountable set of mutually disjoint nonzero components.

REMARK. While $2^{\circ}$ in the above theorem characterizes the $M_{\mu}$ 's in $M$, the analogous property in $L$ is always true, hence cannot characterize the $L_{\mu}$ 's there.

A bounded linear functional $\phi$ on a vector lattice $E$ will be called $\sigma$ continuous if $\lim _{n \rightarrow \infty} \phi\left(a_{n}\right)=\phi(a)$ whenever $a_{n} \rightarrow a$ (that is, $\phi$ preserves sequential convergence). It can be shown by the same argument as was used to prove (3.1) that

(5.3) The $\sigma$-continuous linear functionals on $E$ constitute a closed ideal in $\Omega(E)$. We denote it by $\Omega^{\sigma}(E)$, or simply $\Omega^{\sigma}$.

We have of course $\widetilde{\Omega}(E) \subset \Omega^{\sigma}(E) \subset \Omega(E)$. For the case $E=L, M=\widetilde{\Omega}(L)$ $C \Omega^{\sigma}(L) \subset \Omega(L)=M$, and we have equality. We need not have $L=\Omega^{\sigma}(M)$. For an $L_{\mu}$, however, we have equality in both directions: $M_{\mu}=\tilde{\Omega}\left(L_{\mu}\right) \subset \Omega^{\sigma}\left(L_{\mu}\right)$ $\subset \Omega\left(L_{\mu}\right)=M_{\mu}$, hence $M_{\mu}=\Omega^{\sigma}\left(L_{\mu}\right)$; and, conversely,

(5.4) Given $\mu \in L, L_{\mu}=\widetilde{\Omega}\left(M_{\mu}\right)=\Omega^{\sigma}\left(M_{\mu}\right)$.

Proof. That $L_{\mu}=\tilde{\Omega}\left(M_{\mu}\right)$ follows from the discussion following (4.7). To complete the proof we need only show that every $\sigma$-continuous linear functional on $M_{\mu}$ is continuous. This follows from property $2^{\circ}$ of (5.2).

A closed ideal in $L$ is, as we know, $w(L, M)$-closed. In general, of course, it is not $w(L, C)$-closed; for example, $L_{0}$ is $w(L, C)$-dense in $L$. What about the intermediate topologies, $w(L, S), w(L, B a), w(L, B o)$, and $w(L, U)$ ? These are no better, as the same closed ideal, $L_{0}$, shows. However,

(5.5) Given $\mu \in L, L_{\mu}$ is closed in $L$ under all of the above intermediate topologies.

Proof. Consider $\mu \in L$ and $\nu \notin L_{\mu}$. We show there exists $g \in S \cap B a$ such that $g\left(L_{\mu}\right)=0, g(\nu) \neq 0$, and this will prove the theorem: Let $\rho=|\mu|+|\nu|$; then $\mu \in L_{\rho}, \nu \in L_{\rho}$. Moreover $L_{\mu}$ is a closed ideal in $L_{\rho}$, whence $L_{\rho}=L_{\mu} \oplus L_{\mu}^{\prime}$ and therefore $M_{\rho}=M_{\mu} \oplus M_{\mu}^{\perp}$ (we write $L_{\mu}^{\prime}$ for $L_{\mu}^{\prime}$-in- $L$ and $L_{\mu}^{\perp}$ for $\left(L_{\mu}\right)^{\perp}$-in- $M$ ). Since $\nu \notin L_{\mu}=L_{\mu}^{\perp \perp}$, there exists $f \in L_{\mu}^{\perp}, f>0$ such that $f(\nu) \neq 0$. From [10, (12.3)] there is a sequence $\left\{f_{n}\right\} \subset C_{+}$such that $\left(f_{n}\right)_{p} \rightarrow f$. For each $n$, let $g_{n}=\Lambda_{m>n} f_{m}$. Then $\left(g_{n}\right)_{p}=\Lambda_{m>n}\left(f_{m}\right)_{\rho}$, hence $\left(g_{n}\right)_{\rho} \uparrow f$. It follows $\lim _{n} g_{n}(\nu)$ $=\lim _{n}\left(g_{n}\right)_{\rho}(\nu)=f(\nu)$, and therefore $g_{n}(\nu) \neq 0$ for some $n$. Since $0<\left(g_{n}\right)_{\rho} \leqq f$, we have also that $g_{n}(\omega)=\left(g_{n}\right)_{p}(\omega)=0$ for all $\omega \in L_{\mu}$. Since, finally, $g_{n} \in S \cap B a$, it is the required $g$.

Remark 1. $L_{\mu}$ need not be $w(L, C)$-closed. For example, if $X$ is the real 
interval $0 \leqq x \leqq 1$ and $\mu$ is the Lebesgue measure, then $L_{\mu}$ is separating on $C$, hence $w(L, C)$-dense in $L$.

REMARK 2. It follows trivially from $[10,(6.8)]$ that $L_{1}$ is $w(L, S)$-closed in $L$, hence a fortiori $w(L, B o)$-closed and $w(L, U)$-closed.

6. The weakly compact subsets of $L$. In this section, we characterize the sets in $L$ which are relatively $w(L, M)$-compact. The material here is related to the work of Nakano, Köthe, Dieudonné, and Cooper $[12 ; 6 ; 5]$. We note first that corresponding to the two formulations for absolute continuity of a single element, we have the following for a set.

(6.1) Given $\mu \in L$ and $A \subset L, A$ norm-bounded, the following statements are equivalent:

$1^{\circ}$ The elements of $A$ are equi-uniformly-\| $\|_{\mu}$-continuous on $\Sigma(M)$.

$2^{\circ}$ The elements of $A$ are equi- ||$_{\mu}$-continuous at 0 on $\varepsilon$.

Moreover, in $1^{\circ}$ and $2^{\circ}$ it is enough to consider $A \subset L_{\mu}$ and replace $\Sigma(M)$, $\varepsilon$ by $\Sigma\left(M_{\mu}\right), \varepsilon_{\mu}$ respectively. Also if $1^{\circ}$ and $2^{\circ}$ hold, then they still hold when we replace the elements in $A$ by their absolute values.

If a set $A$ satisfies condition $2^{\circ}$, we will say it is equi-absolutely-continuous with respect to $\mu$.

Proof. We will assume for simplicity that the elements of $A$ have norm $\leqq 1$. Also, given $f \in M, g \in M$, we will denote by $f_{o}$ the component of $f$ in the closed ideal generated by $g$. The following elementary relations are easily verified:

(i) If $e=1,+$, then $f_{e}=f^{+}$.

(ii) If $e=1_{\left(f-\lambda_{1}\right)+}$, then $\lambda e \leqq f_{e}$.

Turning now to the proof itself, $1^{\circ}$ of course implies $2^{\circ}$. Suppose $2^{\circ}$ holds. That it then holds also for the absolute values of the elements of $A$ follows from the quantitative formulation given at the beginning of $\$ 5$. We show next that the absolute values of the elements of $A$ are equi- \|\|$_{\mu}$-continuous at 0 on $\Sigma(M)$.

Suppose not; then there exist $\epsilon>0,\left\{f_{n}\right\} \subset \Sigma\left(M_{+}\right)$, and $\left\{\nu_{n}\right\} \subset A$ such that $\lim _{n}\left\|f_{n}\right\|_{\mu}=0$ while $\left|\nu_{n}\right|\left|f_{n}\right| \geqq 2 \epsilon$ for all $n$. Let $e_{n}=1_{\left(f_{n}-\lambda 1\right)}+, n=1,2, \cdots$. Then from (ii), $\epsilon e_{n} \leqq\left(f_{n}\right)_{e_{n}} \leqq f_{n}$, and therefore $\lim _{n}\left\|e_{n}\right\|_{\mu}=0$. Since the $\left|\nu_{n}\right|$ 's satisfy $2^{\circ}$, this gives $\lim _{n}\left|\nu_{n}\right|\left(e_{n}\right)=0$; and since $\left(f_{n}\right) e_{n} \leqq 1_{e_{n}}=e_{n}$, this gives in turn $\lim _{n}\left|\nu_{n}\right|\left(\left(f_{n}\right) e_{n}\right)=0$. Now $f_{n}-\epsilon 1 \leqq\left(f_{n}-\epsilon 1\right)^{+}=($from $(\mathrm{i}))\left(f_{n}-\epsilon 1\right) e_{n}$ $\leqq\left(f_{n}\right) e_{n}$, or $f_{n} \leqq \epsilon 1+\left(f_{n}\right) e_{n}$, whence

$$
\left|\nu_{n}\right|\left(f_{n}\right) \leqq \epsilon\left|\nu_{n}\right|(1)+\left|\nu_{n}\right|\left(\left(f_{n}\right) e_{n}\right) \leqq \epsilon+\left|\nu_{n}\right|\left(\left(f_{n}\right) e_{n}\right) .
$$

Thus for $n$ large enough, $\left|\nu_{n}\right|\left(f_{n}\right)<2 \epsilon$, which gives a contradiction.

It is now an elementary operation to show that both the elements of $A$ and the absolute values of these elements constitute sets which are equiuniformly \|\|$_{\mu}$-continuous on $\Sigma(M)$. The remainder of the theorem follows easily.

We turn now to relative $w(L, M)$-compactness. For subsets of principal 
closed ideals, the following characterization is known [6, Théorème 4].

(6.2) Given $A \subset L_{\mu}, A$ is relatively $w\left(L_{\mu}, M_{\mu}\right)$-compact (= relatively $w(L, M)$ compact) if and only if it is norm-bounded and equi-absolutely continuous with respect to $\mu$.

Now consider any subset $A$ of $L$. From the Eberlein Theorem [4, Chapter IV, p. 83, Example 15b], $A$ is relatively $w(L, M)$-compact if and only if every countable subset of $A$ is. Since a countable set always lies in some $L_{\mu}$, it follows $A$ is relatively $w(L, M)$-compact if and only if $A \cap L_{\mu}$ is for every $\mu \in L$. We show that $A$ must actually be contained in some $L_{\mu}$.

A set $A$ in a vector lattice will be called normal [6] if $b \in A$ whenever $|b| \leqq|a|$ and $a \in A$. The normal envelope of a set $A$ is the smallest normal set containing $A$; it is easily seen to be the set $\{b|| b|\leqq| a \mid$ for some $a \in A\}$.

(6.3) If a set $A$ in $L$ is relatively $w(L, M)$-compact, its normal envelope is also.

Proof. Denote the normal envelope by $B$. We have to show that $B \cap L_{\mu}$ is relatively $w(L, M)$-compact for every $\mu \in L$. Now $B \cap L_{\mu}$ is precisely the normal envelope of $A \cap L_{\mu}$. Since $A \cap L_{\mu}$ is equi-absolutely continuous with respect to $\mu$ (6.2), it follows from the description of normal evenelope preceding this theorem, that $B \cap L_{\mu}$ is also. Applying (6.2) again gives us the required result.

(6.4) If a set $A$ in $L$ is relatively $w(L, M)$-compact, it lies in some $L_{\mu}$.

Proof. From (6.3), we can assume $A$ is normal. We show every subset $B$ of mutually disjoint nonzero elements of $A$ is countable, whence the theorem will follow easily. Assume $A$ contains an uncountable subset $B$ of mutually disjoint nonzero elements. Then there is an infinite subset $\left\{\mu_{n}\right\}$ of $B$ and a real number $\lambda>0$ such that $\left\|\mu_{n}\right\| \geqq \lambda$ for all $n$. $\left\{\mu_{n}\right\} \subset L_{\mu}$ for some $\mu$, hence it is equi-absolutely-continuous with respect to $\mu$ (6.2). Now for $n \neq m,\left|\mu_{n}\right| \wedge\left|\mu_{m}\right|$ $=0$, hence $\mathbf{1}_{\mu_{n}} \wedge \mathbf{1}_{\mu_{m}}=0$ in $M$. It follows

$$
\sum_{m=1}^{k}\left\|1_{\mu_{m}}\right\|_{\mu}=\left\|\sum_{m=1}^{k} 1_{\mu_{m}}\right\|_{\mu}=\left\|V_{m=1}^{k} 1_{\mu_{m}}\right\|_{\mu} \leqq\|1\|_{\mu},
$$

and therefore $\lim _{m}\left\|1_{\mu_{m}}\right\|_{\mu}=0$. Applying the equi-absolute-continuity of $\left\{\mu_{n}\right\}$, we have that for $m$ large enough, $\left|\mu_{n}\right|\left(1_{\mu_{m}}\right)<\lambda$ for all $n$. This contradicts $\left|\mu_{m}\right|\left(1_{\mu_{m}}\right)=\left\|\mu_{m}\right\| \geqq \lambda$.

With (6.2) and (6.4), we are now in possession of a characterization for relatively $w(L, M)$-compact sets. This is $1^{\circ}$ in the theorem below. We also give two others there. $2^{\circ}$ is due to Nakano $[12,(28.10) ; 1]$; it is obtained also by Cooper [5].

(6.5) $A$ set $A$ in $L$ is relatively $w(L, M)$-compact if and only if it satisfies any one of the following conditions:

1. $A$ is norm-bounded and equi-absolutely-continuous with respect to some $\mu \in L$.

2. If $f_{n} \downarrow 0$ in $M, \lim _{n} f_{n}|\nu|=0$ uniformly on $A$.

3. $A$ is norm-bounded, and any set $B$ of mutually disjoint nonzero elements 
of the normal envelope of $A$ is countable, $B=\left\{\mu_{n}\right\}$, with $\lim _{n}\left\|\mu_{n}\right\|=0$.

Proof. The equivalence of $1^{\circ}$ with relative $w(L, M)$-compactness follows from (6.2) and (6.4). Now let $1^{\circ}$ hold for $A$ and suppose $f_{n} \downarrow 0$ in $M$. We can assume $\left\{f_{n}\right\} \subset \Sigma(M)$. Then since $f_{n} \downarrow 0$ implies $\lim _{n}\left\|f_{n}\right\|_{\mu}=0,2^{\circ}$ follows from (6.1). Let $2^{\circ}$ hold. Since $(1 / n) 1 \downarrow 0$, there exists an $n$, by $2^{\circ}$, such that $|\nu|((1 / n) 1) \leqq 1$ for all $\nu \in A$; hence $\|\nu\|=|\nu|(1) \leqq n$ for all $\nu \in A$, and $A$ is norm-bounded. To prove the second part of $3^{\circ}$, we note first that $2^{\circ}$ clearly holds also for the normal envelope of $A$. Now consider a set $B$ of mutually disjoint elements in this normal envelope, and suppose that for an infinite number of these, $\left\{\mu_{n}\right\},\left\|\mu_{n}\right\| \geqq \lambda>0$. As in (6.4), $\left\{\mu_{n}\right\} \subset L_{\mu}$ for some $\mu$ and $\lim _{m}\left\|1_{\mu_{m}}\right\|_{\mu}=0$. We can assume (since we can take a subsequence, if necessary) that $1_{\mu_{m}} \rightarrow 0$. Since $2^{\circ}$ holds for $\left\{\mu_{n}\right\}$, it follows easily that for $m$ large enough, $\left|\mu_{n}\right|\left(1_{\mu_{m}}\right)<\lambda$ for all $n$. This contradicts $\left|\mu_{m}\right|\left(1_{\mu_{m}}\right)=\left\|\mu_{m}\right\| \geqq \lambda$.

It remains to show that $3^{\circ}$ implies $1^{\circ}$. Let $3^{\circ}$ hold for $A$; we can assume $A$ is normal. It follows from $3^{\circ}$ that $A \subset L_{\mu}$ for some $\mu$. Suppose $A$ is not equiabsolutely-continuous with respect to $\mu$. Then there exists $\lambda>0$ and sequences $\left\{e_{n}\right\} \subset \mathcal{E}_{\mu},\left\{\mu_{n}\right\} \subset A$ such that $\lim _{n}\left\|e_{n}\right\|_{\mu}=0,\left|\mu_{n}\right|\left(e_{n}\right) \geqq \lambda$ for $n=1,2, \cdots$, and $\left|\mu_{n}\right|\left(e_{m}\right)<\lambda / 2^{m}$ for $m>n$ (this last from the individual absolute continuity of $\left.\mu_{1}, \cdots, \mu_{n}\right)$. Let $d_{n}=e_{n}-e_{n} \wedge\left(\mathrm{V}_{m>n} e_{m}\right), n=1,2, \cdots$. Then the $d_{n}$ 's are mutually disjoint components of 1 such that $\lim _{n}\left\|d_{n}\right\|_{\mu}=0$ and $\left|\mu_{n}\right|\left(d_{n}\right) \geqq \lambda / 2$ for $n=1,2, \cdots$.

For each $n$, let $I_{n}$ be the ideal in $M$ generated by $d_{n}$, and $J_{n}$ the dual ideal $\left(I_{n}^{\perp}\right)^{\prime}$-in- $L$. Since $I_{n} \subset M_{\mu}, J_{n} \subset L_{\mu}$ for all $n$; moreover, since for $n \neq m$, $I_{n} \cap I_{m}=0$, we also have $J_{n} \cap J_{m}=0$. Set $\nu_{n}=\left(\mu_{n}\right)_{J_{n}}, n=1,2, \cdots$. Then the $\nu_{n}$ 's are mutually disjoint and $\left|\nu_{n}\right|\left(d_{n}\right)=\left|\mu_{n}\right|\left(d_{n}\right) \geqq \lambda / 2$. Since $\| \nu_{n}||=\left|\nu_{n}\right|\left(d_{n}\right)$, this says $\left\|\nu_{n}\right\| \geqq \lambda / 2$ for all $n$, which contradicts $3^{\circ}$. This completes the proof of (6.5).

Each of the topologies $w(L, U), w(L, B o), w(L, B a), w(L, S)$, and $w(L, C)$ is coarser than $w(L, M)$, and we would therefore expect them to give rise to more relatively compact sets than the latter. And indeed this is true for $w(L, C)$, since the unit ball of $L$ is $w(L, C)$-compact but is not relatively $w(L, M)$-compact. Surprisingly, however,

(6.6) If a subset $A$ of $L$ is relatively compact under one of the topologies $w(L, M), w(L, U), w(L, B o)$, or $w(L, B a)$, then it is relatively compact under all of them.

Proof. It is of course sufficient to prove that if $A$ is relatively $w(L, B a)$ compact, then it is relatively $w(L, M)$-compact. From the discussion following (6.2), we need only show that $A \cap L_{\mu}$ is relatively $w(L, M)$-compact for every $\mu \in L$. Since $w\left(L_{\mu}, M\right)=w\left(L_{\mu}, B a\right)[10,(12.4)]$, we are through.

7. The Mackey topology on $M$. The results of the last section of course enable us to answer the dual problem of characterizing $\tau(M, L)[4$, Chapter IV, §2]. In the following, "topology" will always mean "locally convex topology."

Given a vector lattice $E$, let us consider a topology $T$ on $E$ such that 
(i) $T$ has a neighborhood basis at 0 whose members are (convex and) normal;

(ii) every convergent net also $T$-converges (to the same limit).

If for each member $V$ of this basis, we denote the semi-norm defined on $E$ by \|\|$_{v}$, the above conditions are easily shown to be equivalent to the following: For every $V$ in the basis,

(I) $|a| \leqq|b|$ implies $\|a\|_{v} \leqq\|b\|_{V}$;

(II) $a_{\alpha} \uparrow a$ in $E_{+}$implies $\lim _{\alpha}\left\|a_{\alpha}\right\|_{V}=\|a\|_{V}$;

(III) $a_{\alpha} \downarrow 0$ implies $\lim _{\alpha}\left\|a_{\alpha}\right\|_{V}=0$.

While (II) is superfluous here, Nakano has shown [14, Theorem 6.2] that if $E$ is complete, then in the presence of (II), (III) can be replaced by the weaker condition

(III') $a_{n} \downarrow 0$ implies $\lim _{n}\left\|a_{n}\right\|_{V}=0$.

An example of a topology on $M$ satisfying the above conditions is $|w|(M, L)$. It is the coarsest "normal" (in the sense of (i)) topology which is finer than $w(M, L)[15$, Theorem 6]. We show that the finest topology on $M$ satisfying the conditions is precisely $\tau(M, L)$.

(7.1) $\tau(M, L)$ is identical with the topology defined on $M$ by the set of all semi-norms satisfying (I), (II), and (III').

Proof. Let us denote the latter topology by $T_{0}$. From (ii), every linear functional on $M$ which is $T_{0}$-continuous is continuous, hence lies in $L$. Thus $T_{0} \subset \tau(M, L)$. To show the converse, consider a relatively $w(L, M)$-compact set $A$ in $L$. From (6.3), we can take $A$ to be normal. We have to show that the semi-norm \|\|$_{A^{0}}$ on $M$ defined by the polar $A^{0}$ of $A$ satisfies (I), (II), and

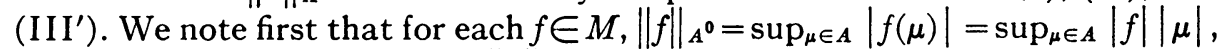
from the normality of $A$. That \|\|$_{A^{0}}$ satisfies (I) is then obvious. Now suppose $f_{\alpha} \uparrow f$ in $M_{+}$. Then $f(\mu)=\sup _{\alpha} f_{\alpha}(\mu)$ for every $\mu \in L_{+}$; hence $\|f\|_{A^{0}=\sup _{\mu \in A} f|\mu|}$ $=\sup _{\mu \in A} \sup _{\alpha} f_{\alpha}|\mu|=\sup _{\alpha} \sup _{\mu \in A} f_{\alpha}|\mu|=\sup _{\alpha}\left\|f_{\alpha}\right\|_{A^{0}}$. Thus \|\|$A_{A^{0}}$ satisfies (II). That \|\|$_{A^{0}}$ satisfies (III') is precisely the content of $2^{\circ}$ in (6.5). This completes the proof. (6.4) gives us

(7.2) Every normal, convex, and closed neighborhood $V$ of 0 in $\tau(M, L)$ has the form $V=\left(V \cap M_{\mu}\right) \oplus M_{\mu}^{\prime}$ for some $\mu \in L$.

Thus $\tau(M, L)$ is determined by the $M_{\mu}$ 's. Also, from (III'),

(7.3) Let $V$ be a neighborhood of 0 in $\tau(M, L)$. If $B$ is any set of mutually disjoint components of 1 , then for only a countable number of these, $\left\{e_{n}\right\}$, do we have $\left\|e_{n}\right\|_{V}>0$; and $\lim _{n}\left\|e_{n}\right\|_{V}=0$.

REMARK 1. It follows from (ii) and $M=\bar{C}$, that $C$ is $\tau(M, L)$-dense in $M$. We also have, either from vector lattice considerations $[18, \S 6]$ or purely vector space properties [4, Chapter III, $\S 3$, Example 18], that $M$ is $\tau(M, L)$ complete.

REMARK 2. Since $|w|(M, L)$ clearly satisfies (i) and (ii), we have $|w|(M, L)$ $C \tau(M, L)$ (cf. also Remark 2 following (4.3)).

REMARK 3. It is obvious that for every $\mu \in L, \tau(M, L)$ is identical on $M_{\mu}$ 
with $\tau\left(M_{\mu}, L_{\mu}\right)$, and that Remarks 1 and 2 remain true if we replace $L, M, C$ by $L_{\mu}, M_{\mu}, C_{\mu}$.

At this point we would like to comment on the role of $|w|\left(L_{\mu}, M_{\mu}\right)$ (the comments could also be made for $\tau\left(M_{\mu}, L_{\mu}\right)$, but $|w|\left(L_{\mu}, M_{\mu}\right)$ has a simpler structure). Let us consider the concrete case where $X$ is the real interval $0 \leqq x \leqq 1$ and $\mu$ is the Lebesgue measure, and we can therefore write $\mathcal{L}^{1}$ for $L_{\mu}$ and $\mathscr{L}^{\infty}$ for $M_{\mu}$. Then the topology $|w|\left(\mathscr{L}^{\infty}, \mathfrak{L}^{1}\right)$ is defined by all the seminorms of the form $\|f\|_{\nu}=\int|f(x)| d \nu$, where $\nu$ is a positive measure absolutely continuous with respect to $\mu$; or equivalently by all the semi-norms of the form $\|f\|_{0}=\int|f(x) g(x)| d \mu$, where $g \in \mathcal{L}^{1}$. This topology is finer than that given by the $\mathscr{L}^{1}$-norm and coarser than that given by the ess-sup norm. Since under the former $\mathscr{L}^{\infty}$ is not complete, and under the latter $C$ is not dense in $\mathscr{L}^{\infty}$, while under $|w|\left(\mathscr{L}^{\infty}, \mathscr{L}^{1}\right)$ we have both $\mathscr{L}^{\infty}$ complete and $C$ dense, this topology might be a more useful tool for studying $\mathscr{L}^{\infty}$ than either of the others.

8. Regular measures. Let $F$ be a sub-vector-lattice of $M$ (not necessarily closed) which contains $C$, and $\pi: L \rightarrow \Omega(F)$ the mapping $\pi \mu=\mu \mid F$. Since $F$ contains $C$, it is separating on $L$. A slight modification of the argument of (2.4) then gives us that $\pi$ is an isomorphism of $L$ onto an ideal in $\Omega(F)$. Finally, the same argument as was used in (3.9) gives us that it is closed. Thus

(8.1) If $F$ is any sub-vector-lattice of $M$ which contains $C$, then $L$ can be identified with a closed ideal in $\Omega(F)$. In particular, this is true for $F=B a, S, B o$, or $U$.

We have characterized $L$ in $\Omega(M)$ as precisely $\tilde{\Omega}(M)$. We now want to characterize $L$ in $\Omega(U), \Omega(B o)$, and $\Omega(B a)$. Let us first confine our attention to $\Omega(U)$. We cannot expect to have an element $\mu$ of $L$ continuous on $U$, since $U$ is not closed and therefore it is possible to have $f_{\alpha} \rightarrow f$ in $U$ and yet $f_{\alpha} \rightarrow f$ in $M$. All we can say is the if $\left\{f_{\alpha}\right\} \subset U, f \in U$, and $f_{\alpha} \rightarrow f$ in $M$, then $\lim _{\alpha} \mu\left(f_{\alpha}\right)$ $=\mu(f)$. It turns out (8.2) that this property does characterize $L$. As (8.2) also shows, this property is equivalent to regularity of $\mu$, which throws some light on the central role regularity has always played.

An element $\phi$ of $\Omega(U)$ will be called a regular $U$-measure, if for each $f \in U$,

$$
|\phi|(f)=\sup _{\sigma \text { uso, }, \leqq f}|\phi|(g)=\inf _{h \text { lsc, } h \geqq f}|\phi|(h) .
$$

(8.2) Given $\phi \in \Omega(U)$, the following statements are equivalent:

1. $\phi \in L$.

2. For every $\left\{f_{\alpha}\right\} \subset U, f \in U, f_{\alpha} \rightarrow f$ in $M$ implies $\lim _{\alpha} \phi\left(f_{\alpha}\right)=\phi(f)$.

3. $\phi$ is regular.

Proof. $1^{\circ}$ of course implies $2^{\circ}$. That $2^{\circ}$ implies $3^{\circ}$ follows from the definition of $U$. We show $3^{\circ}$ implies $1^{\circ}$. We can assume $\phi \geqq 0$. Since $\phi$ is a positive linear functional on $U$, it is also one on $C$, hence coincides on $C$ with an ele- 
ment $\mu$ of $L_{+}$. We show $\phi$ coincides with $\mu$ on all of $U$. Consider $f \in U$. From $[10,(9.6)]$, there exist nets $\left\{g_{\alpha}\right\},\left\{h_{\alpha}\right\},\left\{f_{\alpha}\right\}$, the first consisting of all the u.s.c. elements $\leqq f$ and the second of all the 1.s.c. elements $\geqq f$, such that $g_{\alpha} \uparrow f, h_{\alpha} \downarrow f$, and for each $\alpha, f_{\alpha}$ is an element of $C$ satisfying $g_{\alpha} \leqq f_{\alpha} \leqq h_{\alpha}$. It follows $f_{\alpha} \rightarrow f$ in $M$, hence $\mu(f)=\lim _{\alpha} \mu\left(f_{\alpha}\right)$. Now $\phi$ is regular by hypothesis, hence $\phi(f)=\sup _{\alpha} \phi\left(g_{\alpha}\right)=\inf _{\alpha} \phi\left(h_{\alpha}\right)$; and since $\phi\left(g_{\alpha}\right) \leqq \phi\left(f_{\alpha}\right) \leqq \phi\left(h_{\alpha}\right)$, we have $\phi(f)=\lim _{\alpha} \phi\left(f_{\alpha}\right)$. But $\phi\left(f_{\alpha}\right)=\mu\left(f_{\alpha}\right)$ for all $\alpha$, and thus $\phi(f)=\mu(f)$.

We modify the above argument slightly to obtain

(8.3) An equivalent definition for the regularity of $\phi \in \Omega(U)$ is the following: For each l.s.c. element $f,|\phi|(f)=\sup _{g \in C, o s f}|\phi|(g)$.

Proof. That regularity of $\phi$ implies this condition follows from $1^{\circ}$ in (8.2). Now suppose $\phi$ satisfies the condition, and again we can assume $\phi \geqq 0$. $\phi$ coincides on $C$ with an element $\mu$ of $L_{+}$. It follows immediately from the condition, that $\phi$ coincides with $\mu$ on every l.s.c. element, hence on all of $S$. Now consider $f \in U$. We have of course,

$$
\sup _{\text {ousc, } 0 \leqq f} \mu(g)=\mu(f)=\inf _{h \mid \mathbf{s c}, h \geqq f} \mu(h) .
$$

Since for all such $g, h, \mu(g)=\phi(g) \leqq \phi(f) \leqq \phi(h)=\mu(h)$, (i) gives us

$$
\sup _{0 \text { usc. } g \leqq f} \phi(g)=\phi(f)=\inf _{h \operatorname{lsc}_{\mathbf{s c}}, h \geqq f} \phi(h) .
$$

The major role of $S$ in $U$ is emphasized also in the following generalization of the above (cf. [7, Proposition 6]).

(8.4) If an element $\phi$ of $\Omega(U)$ coincides on $S$ with an element $\mu$ of $L$, and $\|\phi\|=\|\mu\|$, then $\phi$ is identical with $\mu$.

Proof. We show first that the first condition implies $|\phi| \geqq|\mu|$. Consider $f \in U_{+}$and $\epsilon>0$, and choose $g \in S$ such that $0 \leqq g \leqq f,|\mu|(g) \geqq|\mu|(f)-\epsilon$. Turning our attention for a moment to $M_{\mu}$, we can write $M_{\mu}=I \oplus J$ such that $\mu \mid I \geqq 0$ and $\mu \mid J \leqq 0$ (Remark 2 after (3.4)). Choose $h \in S, k \in S$ such that $0 \leqq h_{\mu} \leqq\left(g_{\mu}\right)_{I}, 0 \leqq k_{\mu} \leqq\left(g_{\mu}\right)_{J}$, and $\mu\left(h_{\mu}\right) \geqq \mu\left(\left(g_{\mu}\right)_{I}\right)-\epsilon, \mu\left(k_{\mu}\right) \leqq \mu\left(\left(g_{\mu}\right)_{J}\right)+\epsilon \quad[10$, (12.3), (12.4)]. We can assume that $0 \leqq h \leqq g, 0 \leqq k \leqq g$, and $h \wedge k=0$, because replacing $h, k$ by $h \wedge g, k \wedge g$ does not change $h_{\mu}, k_{\mu}$, and replacing $h, k$ by $h-h \wedge k, k-h \wedge k$ also does not. Then $|\phi|(f) \geqq|\phi|(g) \geqq|\phi|(h \vee k)$ $=|\phi|(h+k) \geqq \phi(h-k)=\mu(h-k)=\mu\left(h_{\mu}\right)-\mu\left(k_{\mu}\right) \geqq \mu\left(\left(g_{\mu}\right)_{I}\right)-\mu\left(\left(g_{\mu}\right)_{J}\right)$ $-2 \epsilon=|\mu|\left(g_{\mu}\right)-2 \epsilon=|\mu|(g)-2 \epsilon \geqq|\mu|(f)-3 \epsilon$. Since $\epsilon$ was arbitrary, this gives $|\phi|(f) \geqq|\mu|(f)$. Thus $|\phi| \geqq|\mu|$. It now follows from $\|\phi\|=\|\mu\|$ that $|\phi|$ $=|\mu|((L)$-space property). Since $L$ is an ideal in $\Omega(U)$, we must have $\phi \in L$, whence $\phi=\mu$.

CoROLlaRy. If an element $\phi$ of $\Omega(U)_{+}$coincides on $S$ with an element $\mu$ of $L$, then $\phi=\mu$.

REMARK. The above two theorems are related to the theory of content $[17, \S \S 53,54]$. 
We can still ask, what are the continuous linear functionals on $U$ ? Since continuity is stronger than regularity, we have $\widetilde{\Omega}(U) \subset L$. We show

$$
\tilde{\Omega}(U)=L_{0} \text {. }
$$

Proof.

Lemma. Given $A \subset U$ and $f \in U, f=\mathrm{V} A$-in- $U$ if and only if $f(x)=\sup _{g \in A} g(x)$ for every $x \in X$.

Assume $f(x)=\sup _{o \in A} g(x)$ for every $x \in X$. This says $f_{0}=\bigvee A_{0}$-in- $M_{0}$, hence $f_{0}=\mathrm{V} A_{0}$-in- $U_{0}$, hence from the isomorphism of $U_{0}$ with $U, f=V A$-in- $U$. Conversely, assume $f=\mathrm{V} A$-in- $U$, and suppose there is a $y \in X$ such that $f(y)$ $-\sup _{o \in A} g(y)=\lambda>0$. Let $h$ be the element in $M_{0}$ defined by $h(y)=\lambda, h(x)=0$ for all $x \neq y$. Then $h \in U[10,(6.8)]$, hence $f-h \in U$. But clearly $f-h \geqq g$ for all $g \in A$, which contradicts $f=V A$-in- $U$.

It follows from the lemma that

(i) if $f_{\alpha} \rightarrow f$ in $U$, then $\lim _{\alpha} f_{\alpha}(x)=f(x)$ for every $x \in X$.

We proceed to prove the theorem. That every $x \in X$ is continuous on $U$ is precisely the statement (i). Thus $L_{0} \subset \widetilde{\Omega}(U)$. We complete the proof by showing that no nonzero element of $L_{1}$ is continuous on $U$. Let $\{\alpha\}$ be the set of finite subsets of $X$ ordered by inclusion, and for each $\alpha$, let $f_{\alpha}$ be the element of $M_{0}$ which is the characteristic function of $\alpha$. Then clearly $f_{\alpha} \uparrow 1_{0}$ in $M_{0}$, hence $f_{\alpha} \uparrow 1_{0}$ in $U_{0}$, hence $f_{\alpha} \uparrow 1$ in $U$. However, for every $\mu \in L_{1}$, $\mu \neq 0, f_{\alpha}|\mu|=0$ for all $\alpha$, while $1|\mu| \neq 0$.

Since $U$ is $\sigma$-closed in $M$, every element of $L$ is $\sigma$-continuous on $U$, and thus $L \subset \Omega^{\sigma}(U)$. Summarizing: $\widetilde{\Omega}(U)=L_{0} \subset\{$ regular $U$-measures $\}=L \subset \Omega^{\sigma}(U)$ $C \Omega(U)$.

We turn to Bo. Defining a regular Borel measure in the same way as a regular $U$-measure, we verify easily that all of the above remains true if we replace $U$ by $B o$. Thus

(8.6) Theorems (8.2)-(8.5) all hold for Bo. And, as for $U, \tilde{\Omega}(B o)=L_{0} \subset\{$ regular Borel measures $\}=L \subset \Omega^{\sigma}(B o) \subset \Omega(B o)$.

When we consider $B a$, the definition of regularity has to be modified, since not all 1.s.c. elements and u.s.c. elements are in $B a$. An element $\phi$ of $B a$ will be called a regular Baire measure if for each $f \in B a$,

$$
|\phi|(f)=\sup _{o \text { usc, }, \in B a, o \leqslant f}|\phi|(g)=\inf _{h \text { lac, } h \in B a, h \geqq f}|\phi|(h) .
$$

We can then obtain the same characterization of $L$ (in $\Omega(B a)$ ) that we have for $U$ and $B o$. However, in this case we have the additional characterization that $L=\Omega^{\circ}(B a)$.

(8.7) Given $\phi \in \Omega(B a)$, the following statements are equivalent:

1. $\phi \in L$.

2. For every $\left\{f_{\alpha}\right\} \subset B a$ and $f \in B a, f_{\alpha} \rightarrow f$ in $M$ implies $\lim _{\alpha} \phi\left(f_{\alpha}\right)=\phi(f)$. 
3. $\phi$ is regular.

4. $\phi$ is $\sigma$-continuous on $B a$.

The proof of this is straightforward once we have the following [17].

(8.8) (1) Every l.s.c. element in Ba is the supremum of a countable subset of $C$, and similarly for every u.s.c. element in $B a$.

(2) Every element of $B a$ is the supremum of the u.s.c. elements in $B a$ below it and the infimum of the l.s.c. elements in Ba above it.

We prove (1). For each $f \in C$, let $I(f)$ be the closed ideal generated in $B a$ by $f$. It is easily verified that $\bigcup_{f \in C} I(f)$ is $\sigma$-closed in $B a$ (cf. the discussion preceding (5.1)), hence in $M$, hence coincides with $B a$. Now consider an l.s.c. element $g$ in $B a$; we can assume $g \geqq 0$. From the above, $g \in I(f)$ for some $f \in C_{+}$. This says $g=\bigvee_{n}(g \wedge n f)$, and since $g \wedge n f$ is in $C$ for each $n,(1)$ is proved. (2) can be proved by the standard type of argument showing that the set of elements described in it is $\sigma$-closed. We omit the details.

We do not go into an examination of $L$ in $\Omega(S)$. The principal reason (perhaps extraneous) for this is that $S$ is in general not norm-closed, and we have not been able to determine what its norm-closure is.

9. The noncontinuous measures. Since $L$ is a closed ideal in $\Omega(M)$, we can write $\Omega(M)=L \oplus L^{\prime}$. Every element of $L$, being continuous on $M$, has its nullideal in $M$ closed. In contrast with this,

(9.1) If $\phi \in L^{\prime}$, the null-ideal of $\phi$ in $M$ is not closed.

Proof. An examination of (3.3) reveals that the following was actually proved: If $\phi \in \Omega(\phi \neq 0)$ has a closed null-ideal in $E$ and is disjoint from an ideal $I$ in $\tilde{\Omega}$, then $\phi$ is not in the $w(\Omega, E)$-closure of $I$. Since the $w(\Omega(M), M)$ closure of $L$ is all of $\Omega(M)$, it follows no nonzero element of $L^{\prime}$ can have a closed null-ideal.

REMARK. For a general element of $\Omega(M)$, the following can be shown:

(9.2) Given $\phi \in \Omega(M)$, the null-ideal of $\phi$ in $M$ is closed if and only if $\phi$ lies in the $w(\Omega(M), M)$-closure of the ideal generated by $\phi_{L}$.

While the $w(\Omega(M), M)$-closure of $L$ is all of $\Omega(M)$, we have

(9.3) The $w(\Omega(M), M)$-closure of $L^{\prime}$ is $L_{1} \oplus L^{\prime}$.

Proof. We will use the following easily verified property of an $(L)$-space $E$ : If $E=I \oplus J$ and $a \in E$, then $a$ is an extreme point of the unit ball of $E$ if and only if it is an extreme point of the unit ball of either $I$ or $J$. Now consider $M_{0}^{\perp}$-in- $\Omega(M)$; we denote it simply by $M_{0}^{\perp} . M_{0}^{\perp}=\left(M_{0}^{\perp} \cap L\right) \oplus\left(M_{0}^{\perp} \cap L^{\prime}\right)$ $=L_{1} \oplus\left(M_{0}^{\perp} \cap L^{\prime}\right)$. By the Krein-Milman theorem [4, Chapter II, $\S 4$ ], $M_{0}^{\perp}$ is the $w(\Omega(M), M)$-closure of the linear subspace generated by the extreme points of its unit ball. But from the property stated at the beginning of the proof, all these extreme points lie in $M_{0}^{\perp} \cap L^{\prime}$; for if any of them were in $L_{1}$, they would also be extreme points of the unit ball of $L$, and all of the latter lie in $X \subset L_{0}\left[9\right.$, Theorem 8]. Thus $L_{1}$ is in the $w(\Omega(M), M)$-closure of $M_{0}^{\frac{1}{1}} \cap L^{\prime}$, hence of $L^{\prime}$.

To complete the proof, we show $L_{1} \oplus L^{\prime}$ is $w(\Omega(M), M)$-closed. Consider 
$\mu \in L_{0}, \mu=\sum_{1}^{\infty} \lambda_{n} x_{n}[10,(4.6)]$, and let $f$ be the element of $M_{0}$ satisfying $f\left(x_{1}\right)=1, f(x)=0$ for all other $x \in X$. Then $\mu(f) \neq 0$, and-by a slight modification of the proof of $[10 ;(6.8)]-\phi(f)=0$ for all $\phi \in \Omega(M)$ disjoint from $\mu$, in particular, for all $\phi \in L_{1} \oplus L^{\prime}$.

We close with a determination of $\left(L^{\prime}\right)^{\perp}$-in- $M$, which we denote simply by $\left(L^{\prime}\right)^{\perp}$. As the last part of the above proof shows, every $f \in M_{0}$ which has value 1 on a single $x$ and 0 on the rest of $X$ lies in $\left(L^{\prime}\right)^{\perp}$; hence of course the normclosed linear subspace generated by such $f$ 's does also. Now an element $g$ of $M_{0}$ lies in this linear subspace if and only if for every $\epsilon>0,|g(x)| \geqq \epsilon$ for only a finite number of $x$ 's. Thus this linear subspace can be identified with $\left(c_{0}\right)$-on- $X$; we will do this. Our result then is

(9.4) $\left(L^{\prime}\right)^{\perp}=\left(c_{0}\right)$-on- $X$.

Proof. For simplicity, we write $\left(c_{0}\right)$. We already have $\left(c_{0}\right) \subset\left(L^{\prime}\right)^{\perp}$; hence $L^{\prime} \subset\left(c_{0}\right)^{\perp}$; hence from (9.3), $L_{1} \oplus L^{\prime} \subset\left(c_{0}\right)^{\perp}$. Since no element of $L_{0}$ is in $\left(c_{0}\right)^{\perp}$, this gives $\left(c_{0}\right)^{\perp}=L_{1} \oplus L^{\prime}$. Then $\left(L^{\prime}\right)^{\perp}=\left(L_{1} \oplus L^{\prime}\right)^{\perp}=\left(c_{0}\right)^{\perp \perp}=\left(c_{0}\right)$.

REMARK. The property $\left(c_{0}\right)^{\perp}=L_{1} \oplus L^{\prime}$ and Banach space theory give us that $L_{0}$ can be identified with $\Omega\left(\left(c_{0}\right)\right)$. Since $L_{0}=l^{1}$-on- $X$, this is of course well-known.

\section{APPENDIX}

10. Absolute continuity. Let $E$ be a vector lattice and $A$ a subset of $\Omega$. As usual, for each $\phi \in \Omega$, we denote by \|\|$_{\phi}$ the semi-norm on $E$ defined by $\|a\|_{\phi}=|\phi||a|$. By $|w|(E, A)$ we will mean the (not necessarily Hausdorff) topology determined on $E$ by the family of semi-norms $\left\{\|\|_{\phi} \mid \phi \in A\right\}$.

(10.1) The set of linear functionals on $E$ which are $|w|(E, A)$-continuous is precisely the ideal I generated by $A$ in $\Omega$.

Proof. Since $\phi \in I$ means $|\phi| \leqq \sum_{1}^{n}\left|\phi_{i}\right|$ for some finite set of elements $\phi_{1}, \cdots, \phi_{n}$ of $A$, it is clear that $|w|(E, A)=|w|(E, I)$. This gives us, in the first place, one half of our theorem, viz. every element of $I$ is $|w|(E, A)$ continuous, and secondly, allows us to use $|w|(E, I)$ in the remainder of the proof. Now suppose $\phi$ is $|w|(E, I)$-continuous on $E$. Then there exist $\psi \in I$ and $\lambda>0$ such that $|\phi(a)| \leqq \lambda\|a\|_{\psi}$ for all $a \in E$. It follows easily that $\phi \in \Omega$ and $|\phi||a| \leqq \lambda|| a \|_{\psi=\lambda|\psi||a|}$ for all $a \in E$. This last says $|\phi| \leqq \lambda|\psi|$ and thus $\phi \in I$.

(10.2) The set of linear functionals on $E$ which are $|w|(E, A)$-continuous on every interval of $E$ is precisely $\bar{I}$.

Proof. As we noted in the previous theorem, $|w|(E, A)=|w|(E, I)$, hence we can assume $A=I$. We first consider the case where $I$ is separating on $E$, and hence $|w|(E, I)$ is Hausdorff. Now each interval $[-a, a](a>0)$ in $E$ is $|w|(E, I)$-closed. Also $\bar{I}$ is the completion of $I$ under $|w|(\Omega, E)[10,(11.5)]$. Since the latter is the topology of uniform convergence on intervals of $E$, the Grothendieck theorem [4, Chapter IV, §3, Example 3b] gives us our desired conclusion. 
Now suppose $I$ is not separating on $E$. $I^{\perp}$ (in $E$ ) is an ideal, hence $E / I^{\perp}$ is a vector lattice under the usual definition: given $B \in E / I^{\perp}, B \geqq 0$ if $b \geqq 0$ for some $b \in B$. Since $E / I^{\perp}$ is separating on $I, I$ can be considered a subset of $\Omega\left(E / I^{\perp}\right)$. Moreover, each interval $[-B, B](B>0)$ of $E / I^{\perp}$ has the form $[-b, b]+I^{\perp}$. It follows $|w|\left(I, E / I^{\perp}\right)=|w|(I, E)$, and therefore $\bar{I}$ is the $|w|\left(I, E / I^{\perp}\right)$-completion of $I$. The Grothendieck theorem then again concludes the argument.

Corollary. Let $E$ be a vector lattice and $\Omega$ be separating on $E$. Then the set of linear functionals on $\Omega$ which are $|w|(\Omega, E)$-continuous on every interval of $\Omega$ is precisely $\tilde{\Omega}(\Omega)$.

Simply let $\Omega, \Omega(\Omega), E$ be the $E, \Omega, A$ of the above theorem and apply (3.7).

\section{BIBLIOGRAPHY}

1. I. Amemiya, On the equi-continuity in semi-ordered linear spaces, Proc. Japan Acad. vol. 27 (1951) p. 275.

2. G. Birkhoff, Lattice theory, Amer. Math. Soc. Colloquium Publications, 1940, rev. ed. 1948.

3. N. Bourbaki, Integration, Paris, Hermann et Cie., Chapters I-IV, 1952.

4. - Espaces vectoriels topologiques, Paris, Hermann et Cie., Chapters I-V, 1953 and 1955.

5. J. L. B. Cooper, Coordinated linear spaces, Proc. London Math. Soc. Ser. 3 vol. 3 (1953) pp. 305-327.

6. J. Dieudonné, Sur les espaces de Köthe, J. Analyse Math. vol. 1 (1951) pp. 81-115.

7. - Sur la convergence des suites de measures de Radon, An. Acad. Brasil. Ci. vol. 23 (1951) pp. 21-38.

8. S. Kakutani, Concrete representation of abstract $(L)$-spaces and the mean ergodic theorem, Ann. of Math. vol. 42 (1941) pp. 523-537.

9. - Concrete representation of abstract (M)-spaces, Ann. of Math. vol. 42 (1941) pp. 994-1024.

10. S. Kaplan, The second dual of the space of continuous functions, Trans. Amer. Math. Soc. vol. 86 (1957) pp. 70-90.

11. M. Nakamura, Notes on Banach space (X), Tôhoku Math. J. vol. 1 (1949-1950) pp. 100-108.

12. H. Nakano, Modulared semi-ordered linear spaces, Tokyo, Maruzen Co., 1950.

13. - Stetige lineare Functionale auf dem teilweise geordneten Modul, J. Fac. Sci. Imp. Univ. Tokyo vol. 4 (1942) pp. 201-382.

14. - Linear topologies on semi-ordered linear spaces, J. Fac. Sci. Hokkaido Univ. vol. 12 (1953) pp. 87-104.

15. G. T. Roberts, Topologies in vector lattices, Proc. Cambridge Philos. Soc. vol. 48 (1952) pp. 533-546.

16. I. Namioka, Partially ordered linear topological spaces, Memoirs Amer. Math. Soc., no. 24, 1957, $50 \mathrm{pp}$.

17. P. R. Halmos, Measure theory, New York, D. van Nostrand, 1950.

18. I. Amemiya and T. Mori, Topological structures in ordered linear spaces, J. Math. Soc. Japan vol. 9 (1957) pp. 131-142.

INSTITUTE FOR AdVANCED STUdy, Princeton, New Jersey

Wayne State University, Detroit, Michigan 